\title{
Super-resolution X-ray holography
}

\author{
J. Soltau, M. Vassholz, M. Osterhoff, T. Salditt \\ Institut für Röntgenphysik, Georg-August-Universität Göttingen, Friedrich-Hund-Platz 1, Göttingen, Germany
}

\author{
jakob.soltau@uni-goettingen.de
}

For non-destructive imaging of three-dimensional (3D) materials and biological specimen, hard X-ray in-line holography is particularly suitable, since it offers a phase-sensitive imaging scheme which can cover large specimen in a full-field approach without the need for scanning. Unfortunately, the resolution of holographic imaging is limited by the source size of the cone-beam illumination, and does not reach values in the sub-20 nm regime which are routinely achieved by ptychography or CDI.

We have implemented holographic X-ray imaging based on cone-beam illumination, beyond the resolution limit given by the conebeam numerical aperture. In this new single-shot approach [1], image information encoded in the far-field diffraction and in the holographic self-interference is treated in a common reconstruction scheme, without the usual empty beam correction step of in-line holography. An illumination profile tailored by waveguide optics and exactly known by prior ptychographic probe retrieval is shown to be sufficient for solving the phase problem. We demonstrate the improved experimental capability by reconstruction of a test pattern with a field of view of $5 \times 5 \mu \mathrm{m}^{2}$ and a resolution of $11 \mathrm{~nm}$, using a waveguide exit source size of about $30 \mathrm{~nm}$ (FWHM).

Figure 1 shows the divergent illumination (a) from the waveguide exit (b) to the sample plane (c). To quantify the resolution we have analyzed the reconstruction of a pattern with $50 \mathrm{~nm}$ (half-period) lines and spaces (d,e). The reconstruction by the presented method shows higher resolution and image quality compared to conventional single-shot reconstruction by the contrast-transfer-function approach after empty-beam division. The resolution of the new reconstruction approach was determined by Fourier ring correlation (FRC) indicating a resolution (half-period) of $\Delta=11.2 \mathrm{~nm}$ (f).

The approach paves the way towards high resolution and dose-efficient X-ray tomography, well suited for the current upgrades of synchrotron radiation sources to diffraction limited storage rings.

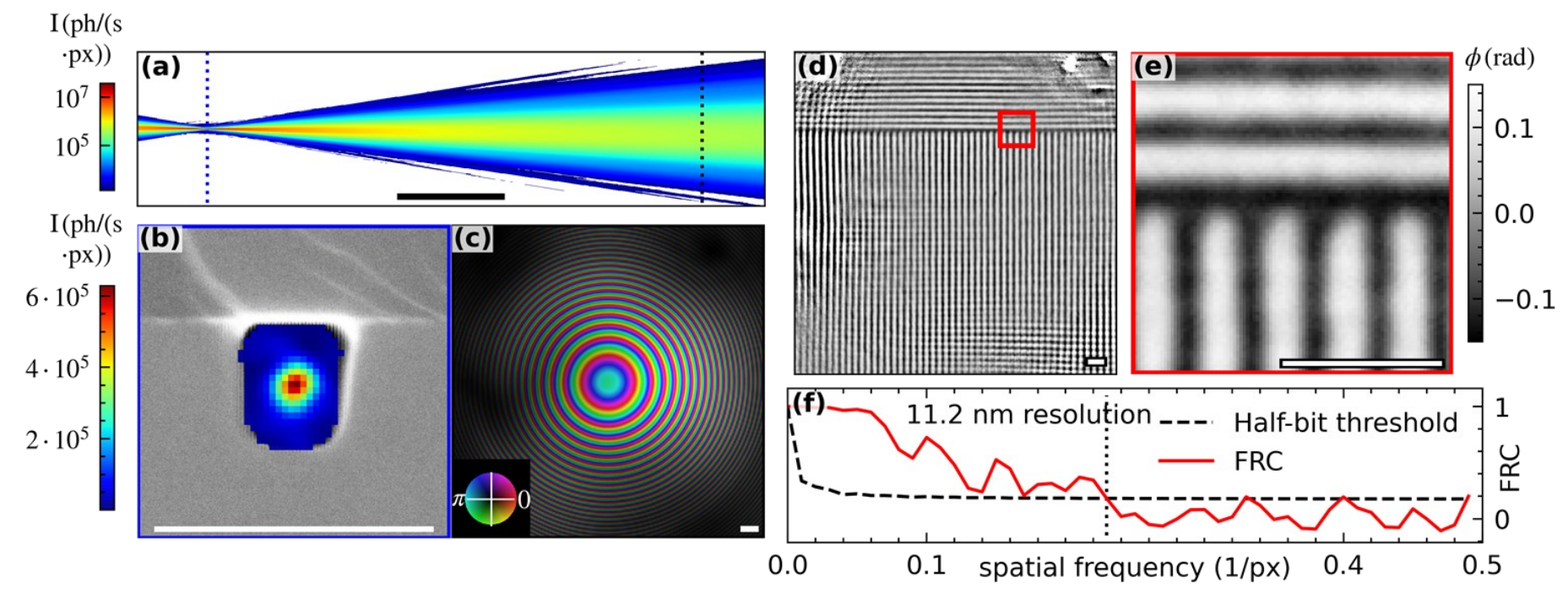

Figure 1. (a) reconstructed and propagated waveguide intensity, the dotted blue line indicates the waveguide exit plane, the dotted black line indicates the sample plane. (b) simulated intensity distribution leaving the waveguide, resulting in a source spot with a FWHM of $29 \times 32 \mathrm{~nm}{ }^{2}$. (c) phase and amplitude of the wave-field in the sample plane reconstructed by ptychography. (d) shows the reconstructed $50 \mathrm{~nm}$ lines and spaces structure from a single shot detector image. (e) magnified region of interest (ROI). (f) FRC analysis of (e), correlating reconstructions of two independent measurements with an acquisition time of $2 \mathrm{~s}$ each, indicating a resolution (half-period) of $\Delta=11.2 \mathrm{~nm}$. Scalebars: (a) $250 \mu \mathrm{m}$, (b-e) $250 \mathrm{~nm}$.

[1] J. Soltau, M. Vassholz, M. Osterhoff and T. Salditt, Inline holography with hard X-rays at sub-15 nm resolution, (in review).

Keywords: Single-shot, Holography, Ptychography, Near-field imaging, X-ray optics 